\title{
Neutrophil/lymphocyte ratio is a more sensitive systemic inflammatory response biomarker than platelet/lymphocyte ratio in the prognosis evaluation of unresectable pancreatic cancer
}

\author{
Yuan Gao ${ }^{1, *}$, Wen-Jie Wang ${ }^{1, *}$, Qiaoming Zhi' ${ }^{2,}{ }^{,}$, Meng Shen ${ }^{1}$, Min Jiang ${ }^{1}$, Xiaojie \\ Bian $^{1}$, Fei-Ran Gong ${ }^{3}$, Chong Zhou ${ }^{4}$, Lian Lian ${ }^{1,5}$, Meng-Yao Wu ${ }^{1}$, Jun Feng ${ }^{1}$, Min \\ $\mathrm{TaO}^{1,6}$ and Wei $\mathbf{L i}^{1,6,7}$ \\ ${ }^{1}$ Department of Oncology, The First Affiliated Hospital of Soochow University, Suzhou, China \\ ${ }^{2}$ Department of General Surgery, The First Affiliated Hospital of Soochow University, Suzhou, China \\ ${ }^{3}$ Department of Hematology, The First Affiliated Hospital of Soochow University, Suzhou, China \\ ${ }^{4}$ Department of Radiation Oncology, Xuzhou Central Hospital, Xuzhou, China \\ ${ }^{5}$ Department of Oncology, Suzhou Xiangcheng People's Hospital, Suzhou, China \\ ${ }^{6}$ PREMED Key Laboratory for Precision Medicine, Soochow University, Suzhou, China \\ ${ }^{7}$ Center for Systems Biology, Soochow University, Suzhou, China \\ *These authors have contributed equally to this work \\ Correspondence to: Wei Li, email: liwei10@suda.edu.cn \\ Meng-Yao Wu, email: mywu@suda.edu.cn \\ Jun Feng, email: fengjun2001-260@163.com \\ Keywords: pancreatic cancer, systemic inflammatory response (SIR), neutrophil to lymphocyte ratio (NLR), platelet to lymphocyte \\ ratio (PLR) \\ Received: April 21,2017 Accepted: July 26, $2017 \quad$ Published: September 27, 2017 \\ Copyright: Gao et al. This is an open-access article distributed under the terms of the Creative Commons Attribution License 3.0 \\ (CC BY 3.0), which permits unrestricted use, distribution, and reproduction in any medium, provided the original author and source \\ are credited.
}

\section{ABSTRACT}

Multiple cancers arise from sites of infection, chronic irritation, and inflammation. It has been widely accepted that pancreatic cancer is an inflammation-driven cancer. In this study, we investigated the application value of systemic inflammatory markers, neutrophil to lymphocyte ratio (NLR) and platelet to lymphocyte ratio (PLR), in the prediction of chemotherapy response and prognosis in patients with late pancreatic cancer. 122 patients with inoperable pancreatic cancers were included and separated into two groups according to median values of NLR or PLR (NLR low: < 3.81 or NLR high: $\geq 3.81$, and PLR low: $<142.14$ or PLR high $\geq 142.14$, respectively). Baseline NLR and PLR levels were significantly higher in pancreatic cancer patients compared with the healthy subjects. Neither of the baseline NLR or PLR levels could predict outcomes. Patients with low baseline level of NLR, but not PLR, had better responses to chemotherapy. Changes in NLR, but not PLR levels, were associated with the therapeutic efficacy. Patients who stayed in or dropped into the low NLR level subgroup after first-line chemotherapy had better responses, comparing to those stayed in or jumped into the high NLR level group. No similar results could be observed when the PLR level was investigated. Therefore, NLR is a more sensitive biomarker than PLR in the prediction of chemotherapy response of patients. 


\section{INTRODUCTION}

Pancreatic cancer is an extremely malignant solid cancer with a collective 1-year survival of just $26 \%$, and 5 -year survival less than 5\%. Almost all of the patients have lost the chance of surgery at the time of diagnosis. Even for those who received surgery, they might finally suffer from recurrence or metastasis. Chemotherapy is still one of the major treatments for pancreatic cancer [1] [2] [3].

Systemic inflammatory response (SIR) is reported to be closely related to outcomes in many cancers, including pancreatic cancer [4]. It has been proved that neutrophil, lymphocyte and platelet levels in peripheral venous blood could be affected by tumor-induced SIR [5]. Therefore, the quantifications of these hematological parameters have been analyzed as markers of SIR in various malignant tumors, including pancreatic cancer $[6,7]$.

Researches have found that both platelet to lymphocyte ratio (PLR) and neutrophil to lymphocyte ratio (NLR) have predictive value in early diagnosis, and can reflect prognosis to some extend. It is reported that elevated levels of NLR usually suggested poor prognosis in patients with colorectal cancer who underwent primary resection or hepatectomy for liver metastases $[6,8]$. PLR is regarded as a biomarker combining the apparent pre-inflammatory and prophylactic status of cancer with endogenous residual cancer resistance [9].

NLR and PLR have been proved to be both reliable and cost-effective [10], giving them promising prospects in the diagnosis and treatment of cancers. However, the sensitivities of NLR and PLR have rarely been compared in pancreatic cancer. In our study, we tried to evaluate the value of PLR and NLR in chemotherapy response and outcomes of patients with advanced pancreatic cancer.

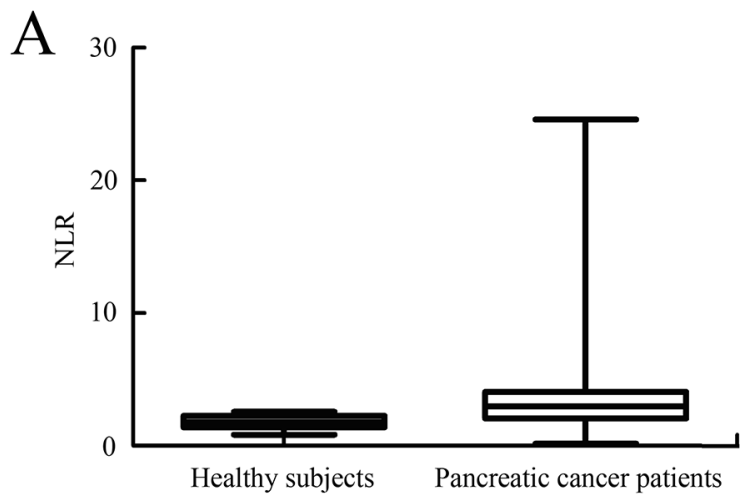

\section{RESULTS}

\section{Baseline levels of NL\R and PLR were higher in patients with pancreatic cancer comparing with the controls}

The mean baseline NLR level of pancreatic cancer patients was $3.81 \pm 3.65$, and the corresponding level of healthy subjects was $1.80 \pm 0.48$. Patients with pancreatic cancer had a significant higher baseline level of NLR than the controls $(P<0.001)$ (Figure 1A). The mean baseline PLR level of pancreatic cancer patients was $142.14 \pm 86.76$, while the corresponding level of healthy subjects was $112.34 \pm 25.52$. Pancreatic cancer patients also had a higher baseline PLR level comparing with the controls $(P<0.001)$ (Figure 1B). Increased baseline NLR and PLR values in pancreatic cancer group suggested that these biomarkers could be used to differentiate pancreatic cancer patients from healthy controls and might be used in the early diagnosis of pancreatic cancer.

As presented in Table 1, high baseline PLR and NLR levels both correlated with smoking history. High baseline level of PLR was related to liver metastasis as well.

\section{Relationship between baseline NLR level and baseline PLR level}

To investigate whether baseline NLR level and baseline PLR level were associated, patients were divided in to low and high PLR or NLR goups, according to baseline PLR or NLR levels respectively. As shown in Figure 2A, NLR levels of patients in high PLR group were higher than those in low PLR group $(P<0.001)$. Likewise, PLR levels of patients in high NLR group were higher than those in low NLR group $(P<0.001$, Figure 2B).

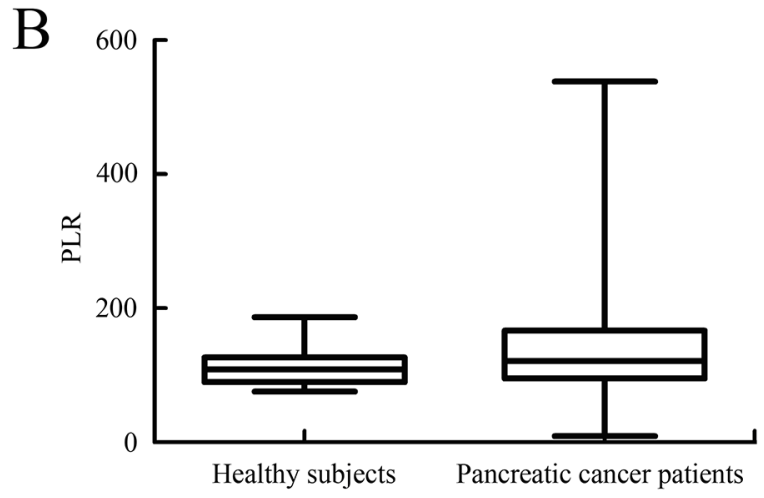

Figure 1: Comparation of baseline NLR and PLR values in pancreatic cancer group with the ones in healthy subjects. (A) Comparation of baseline NLR values in pancreatic cancer group with the ones in healthy subjects. Baseline NLR level was significantly higher in pancreatic cancer patients than in the healthy subjects $(P<0.001)$. (B) Comparation of baseline PLR values in pancreatic cancer group with the ones in healthy subjects. Baseline PLR level was significantly higher in pancreatic cancer patients comparing with the healthy subjects $(P<0.001)$. 
Table 1: Relationship between baseline NLR, PLR level and clinicopathological features

\begin{tabular}{|c|c|c|c|c|c|c|c|c|c|}
\hline \multirow{2}{*}{$\begin{array}{l}\text { Clinicopathologic } \\
\text { features }\end{array}$} & \multirow[t]{2}{*}{ n } & \multicolumn{4}{|c|}{ NLR } & \multicolumn{4}{|c|}{ PLR } \\
\hline & & Low (n) & High (n) & $\chi^{2}$ & $P$ value & Low (n) & High (n) & $\chi^{2}$ & $P$ value \\
\hline \multicolumn{10}{|l|}{ Gender } \\
\hline Men & 74 & 40 & 34 & 1.788 & 0.181 & 34 & 40 & 0.787 & 0.375 \\
\hline Women & 48 & 20 & 28 & & & 26 & 22 & & \\
\hline \multicolumn{10}{|l|}{ Age (years) } \\
\hline$<65$ & 58 & 26 & 32 & 0.838 & 0.360 & 28 & 30 & 0.036 & 0.849 \\
\hline$\geq 65$ & 64 & 34 & 30 & & & 32 & 32 & & \\
\hline \multicolumn{10}{|l|}{$\begin{array}{l}\text { BMI (Body mass } \\
\text { index) }\end{array}$} \\
\hline$<25$ & 60 & 28 & 32 & 0.298 & 0.585 & 29 & 31 & 0.034 & 0.854 \\
\hline$\geq 25$ & 62 & 32 & 30 & & & 31 & 31 & & \\
\hline \multicolumn{10}{|l|}{ Smoke } \\
\hline No & 53 & 35 & 18 & 10.655 & $0.001 * *$ & 32 & 21 & 4.701 & $0.030 *$ \\
\hline Yes & 69 & 25 & 44 & & & 28 & 41 & & \\
\hline \multicolumn{10}{|l|}{ Liver metastasis } \\
\hline No & 43 & 29 & 14 & 8.860 & $0.003 * *$ & 20 & 23 & 0.189 & 0.664 \\
\hline Yes & 79 & 31 & 48 & & & 40 & 39 & & \\
\hline
\end{tabular}

Therefore, baseline NLR level and baseline PLR level correlated with each other.

\section{Baseline NLR level predicted chemotherapeutic efficacy}

The relationships between baseline PLR or NLR levels and chemotherapeutic efficacy were presented in Table 2 and Table 3. The value of baseline NLR correlated with chemotherapeutic efficacy. However, baseline PLR level was not associated with chemotherapeutic efficacy.

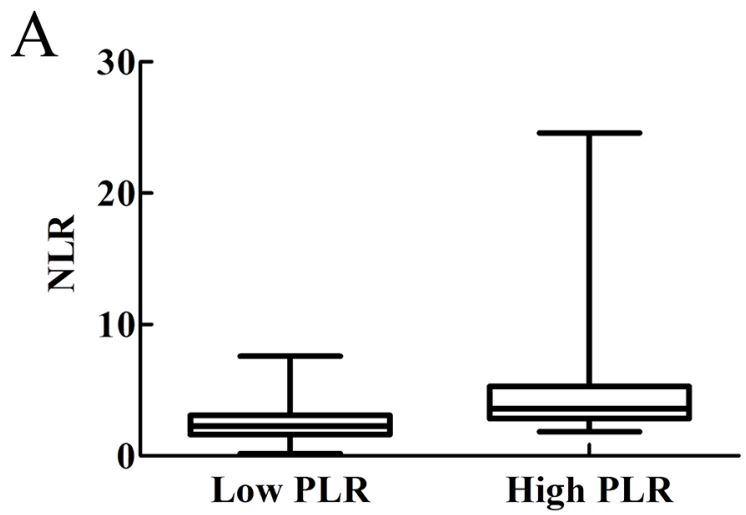

\section{Baseline PLR and NLR levels were not related to outcomes}

The median OS of all patients was 10 months (9.7310.27 months) (Figure 3A). Survivors were followed for 25 months. We used the Kaplan-Meier diagrams to evaluate the impact of the PLR and NLR states on OS (Figure 3B and 3C). The median OS was 10 months (9.5210.48 ) for high NLR group, and 10 months (9.68-10.32) for the low NLR group respectively $(P>0.05)$. The median OS of the high PLR group was 10 months (9.65-10.35),

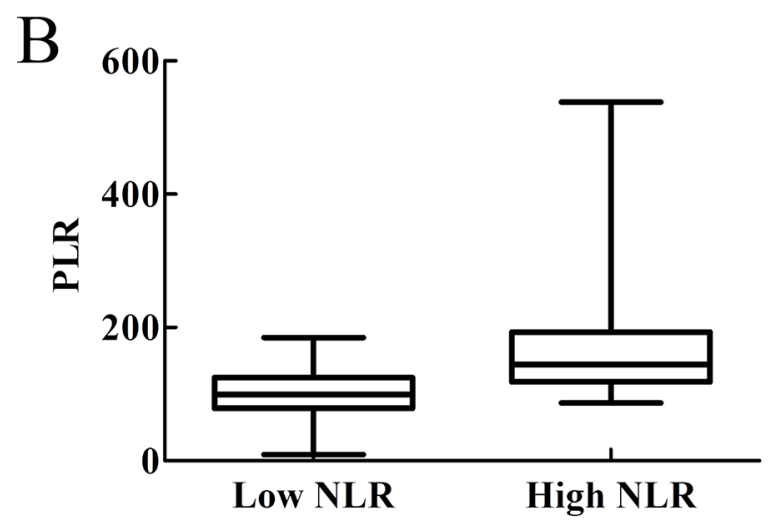

Figure 2: Relationship between baseline NLR level and baseline PLR level. (A) Patients were divided into low and high PLR groups. NLR level of patients in high PLR group were higher than that of subjects in low PLR group $(P<0.001)$. (B) Patients were divided into low and high NLR groups. PLR level of patients in high NLR group were higher than that of subjects in low NLR group $(P<0.001)$. 
Table 2: Relationship between NLR baseline levels and chemotherapeutic efficacy

\begin{tabular}{lcccc}
\hline NLR levels & PR+SD $(\mathbf{n}=\mathbf{7 6})$ & PD $(\mathbf{n}=\mathbf{4 6})$ & $\boldsymbol{\chi 2}$ & $\boldsymbol{P}$ value \\
\hline Low $(\mathrm{n}=60)$ & 47 & 13 & 12.929 & $<0.001$ \\
High $(\mathrm{n}=62)$ & 29 & 33 & & \\
\hline
\end{tabular}

PR: partial response; SD: stable disease; PD: progressive disease.

Table 3: Relationship between PLR baseline levels and chemotherapeutic efficacy

\begin{tabular}{lcccc}
\hline PLR levels & PR+SD $(\mathrm{n}=\mathbf{7 6})$ & $\mathbf{P D}(\mathrm{n}=\mathbf{4 6})$ & $\boldsymbol{\chi 2}$ & $\boldsymbol{P}$ value \\
\hline Low $(\mathrm{n}=60)$ & 33 & 27 & 2.675 & 0.102 \\
High $(\mathrm{n}=62)$ & 43 & 19 & & \\
\hline
\end{tabular}

Table 4: Relationship between changes in NLR level and chemotherapeutic efficacy

\begin{tabular}{|c|c|c|c|c|c|}
\hline Pre-chemotherapy & Post-chemotherapy & $P R+\operatorname{SD}(n=76)$ & $\operatorname{PD}(n=46)$ & $\chi 2$ & $P$ value \\
\hline \multirow[t]{2}{*}{ Low $(n=60)$} & Low $(n=42)$ & 36 & 6 & 4.494 & 0.034 \\
\hline & High $(n=18)$ & 11 & 7 & & \\
\hline \multirow[t]{2}{*}{$\operatorname{High}(n=62)$} & Low $(n=16)$ & 11 & 5 & 4.183 & 0.041 \\
\hline & $\operatorname{High}(n=46)$ & 18 & 28 & & \\
\hline
\end{tabular}

and the low PLR group was 10 months (9.65-10.35) $(P>0.05)$. Therefore, baseline PLR and NLR levels were not correlated with outcomes.

\section{Changes in NLR level after chemotherapy were related to chemotherapeutic efficacy}

To identify the relationship between changes of PLR or NLR levels and chemotherapeutic efficacy, blood samples were obtained and computed tomography (CT) scan assessments were performed at the same time subsequent to first-line chemotherapy. After chemotherapy, 42 patients with low baseline NLR levels retained (Table 4), while 18 patients changed to high NLR group. 46 patients with high baseline NLR levels reserved in this group. In contrast, 16 patients with high baseline NLR levels changed to low NLR group. Patients with low NLR levels after first-line chemotherapy (no matter remained or transferred to) had improved responses comparing with those retained or were transferred to high NLR group.

After first-line chemotherapy, 30 patients were still reserved in low baseline PLR level group (Table 5), while 30 patients were transferred to high PLR group. Meanwhile, 34 patients in high baseline PLR level group maintained in this group, and 28 patients from this group changed to low PLR level group. Patients with low PLR
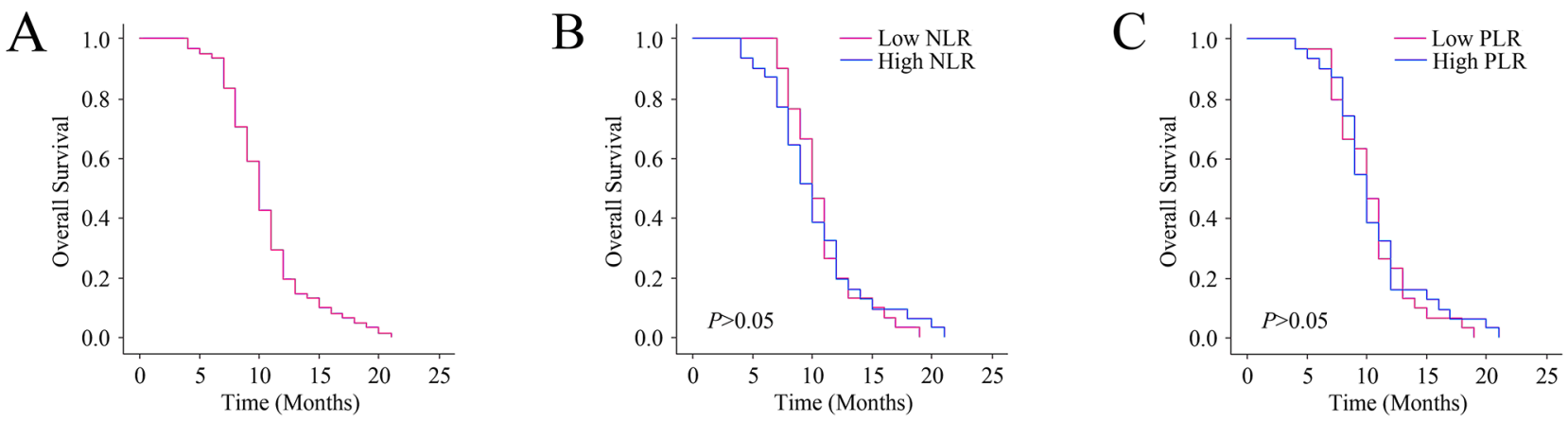

Figure 3: Relationship between baseline NLR and PLR levels and the outcomes. (A) predicted probability of overall survival (OS). (B) The OS according to NLR. (C) The OS according to PLR. 
Table 5: Relationship between changes in PLR level and chemotherapeutic efficacy

\begin{tabular}{|c|c|c|c|c|c|}
\hline Pre-chemotherapy & Post-chemotherapy & $P R+S D(n=76)$ & $\operatorname{PD}(n=46)$ & $\chi^{2}$ & $P$ value \\
\hline \multirow[t]{2}{*}{ Low $(n=60)$} & Low $(n=30)$ & 18 & 12 & 0.606 & 0.436 \\
\hline & High $(n=30)$ & 15 & 15 & & \\
\hline \multirow[t]{2}{*}{ High $(n=62)$} & Low $(n=28)$ & 21 & 7 & 0.766 & 0.382 \\
\hline & $\operatorname{High}(n=34)$ & 22 & 12 & & \\
\hline
\end{tabular}

levels after first-line chemotherapy (no matter remained or transferred to) did not exhibit improved responses comparing with those remained or transferred to high PLR level group (Table 5).

Therefore, changes in NLR level, but not PLR level, after chemotherapy associated with chemotherapeutic efficacy.

\section{Changes in NLR level predicted outcomes}

The Kaplan-Meier diagrams were performed to evaluate the impact of changes in the PLR or NLR levels on OS (Figure 4). The median OS was 10 (9.77-10.23) months for patients with elevated NLR levels after first-line chemotherapy, comparing with 11 months (10.48-11.52) for NLR-decreased patients $(P<0.001)$. The median OS of PLR-increased group was 10 months (9.65-10.35), and the PLR-decreased group was 10 months (9.61-10.39) $(P>0.05)$. As a result, the survival rates of patients with elevated NLR levels after chemotherapy is reduced. But the changes in PLR level did not show prognostic value.

To evaluate the factors which were related to OS, univariate and multivariate analyses were further performed. By using the post-/pre-chemotherapy PLR or NLR ratios, patients were separated into two groups respectively. Post-/pre-chemotherapy ratios $<1$ represented that the values of NLR or PLR were decreased after chemotherapy, and $\geq 1$ implied that PLR or NLR values were not decreased. As shown in Table 6, univariate and multivariate analysis confirmed that post/prechemotherapy NLR ratio is a prognostic factor affecting OS. Therefore, changes in NLR level, but not PLR level, after chemotherapy predicted outcomes.

\section{DISCUSSION}

Inflammation, as a major feature of tumors [11], has been proved to be a crucial and essential process in the progression of malignancies [12] [13], including proliferation, angiogenesis, metastasis and chemotherapyresistance [11]. Pancreatic cancer has a rather unique and complex microenvironment which is rich in inflammatory cytokines, growth factors and proteinases that are favorable for proliferation, invasion and metastasis of pancreatic cancer cells [14] [15]. Increased serum levels of inflammatory cytokines are also related to clinical features of pancreatic cancer, such as poor performance status, cachexia and OS [16, 17]. Therefore, cancer-associated inflammation is a key molecular feature in pancreatic cancer.

Systemic inflammation is considered to be able to predict poor outcomes among several types of malignances, including pancreatic cancer [18]. So far, lots of systemic inflammatory markers have been found to have predictive value for prognosis $[19,20]$. For example, as a SIR marker, incorporating C-reactive protein (CRP),

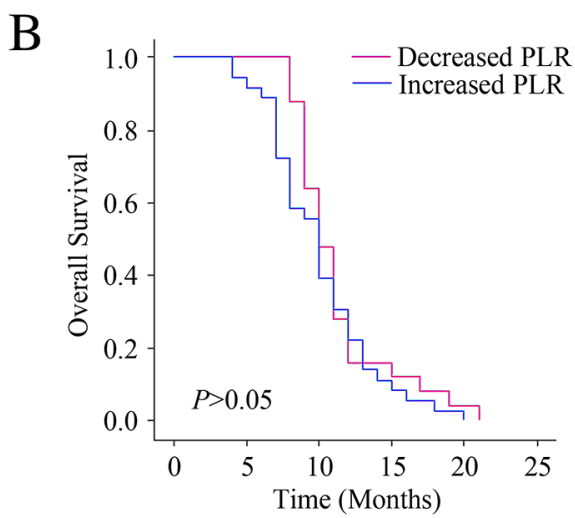

Figure 4: Relationship between changes in NLR and PLR levels after chemotherapy and the outcomes. (A) The OS according to changes in NLR. (B) The OS according to changes in PLR. 
Table 6: Univariate and multivariate analysis of risk factors for the overall survival

\begin{tabular}{|c|c|c|c|c|}
\hline \multirow[t]{3}{*}{ Risk factors } & \multicolumn{4}{|c|}{ Overall survival (OS) } \\
\hline & \multicolumn{2}{|c|}{ Univariate analysis } & \multicolumn{2}{|c|}{ Multivariate analysis } \\
\hline & OR $(95 \%$ CI $)$ & $P$ value & OR $(95 \%$ CI $)$ & $P$ value \\
\hline $\begin{array}{l}\text { Gender } \\
\text { (Women or Men) }\end{array}$ & $0.770(0.528-1.124)$ & 0.175 & - & - \\
\hline $\begin{array}{l}\text { Age } \\
(>65 \text { years or } \leq 65 \text { years })\end{array}$ & $0.773(0.537-1.110)$ & 0.163 & - & - \\
\hline $\begin{array}{l}\text { PLR } \\
\text { (high or low) }\end{array}$ & $0.922(0.641-1.325)$ & 0.660 & - & - \\
\hline $\begin{array}{l}\text { NLR } \\
\text { (high or low) }\end{array}$ & $1.007(0.701-1.448)$ & 0.969 & - & - \\
\hline $\begin{array}{l}\text { Post/pre-chemotherapy PLR ratio } \\
(>1 \text { or } \leq 1)\end{array}$ & $1.272(0.881-1.837)$ & 0.199 & - & - \\
\hline $\begin{array}{l}\text { Post/pre-chemotherapy NLR ratio } \\
(>1 \text { or } \leq 1)\end{array}$ & $1.902(1.290-2.803)$ & $0.001 * *$ & $1.902(1.290-2.803)$ & $0.001 * *$ \\
\hline
\end{tabular}

together with serum albumin, has a close relationship with outcomes in cancer patients [9]. Besides, serum lactate dehydrogenase (LDH) levels are also associated with the systemic inflammatory response and serves as a significant prognostic predictor in pancreatic cancer [21]. Systemic inflammation can also induce measurable neutrophilia and relative lymphocytopenia in the peripheral blood [19].

Neutrophils play an active role in both systemic and local inflammatory response. It is believed that up-regulation of neutrophils can reflect an aggressive feature of cancer cells since it is primarily stimulated by hematopoietic cytokines derived from tumor cells [22]. Neutrophils can produce arginase, nitric oxide (NO), reactive oxygen species (ROS), and inhibit the function of cytotoxic lymphocytes [23, 24]. In addition, preclinical researches have showed that neutrophils functioned as tumor-promoting factors via the signaling pathway triggered by transforming growth factor $\beta$ (TGF- $\beta$ ) [25].

Lymphocytes, on the other hand, possess the anti-cancer activities through repressing tumor growth or metastasis [26]. Lymphocyte counts can reflect endogenous cancer resistance of the immune system [27, 28 ]. It has been proved that higher number of lymphocytes predicts better outcomes [27, 28]. Elevated numbers of lymphocytes might be related to a tumor shrink after neoadjuvant chemoradiotherapy in locally advanced rectal cancer [29].

By the combination of neutrophils and lymphocytes, high NLR level has been shown to be a promising indicator of poor prognosis for patients with resectable colorectal cancer [6]. Elevated NLR level is suggested as a marker for estimating benefits stage II colon cancer patients obtained from adjuvant treatment, and could identify risks of those patients [30]. Baseline NLR measurements and changes in NLR levels after therapy also have predictive values in outcomes of patients with unresectable gastric cancer [31]. Besides, NLR was also useful in determining prognosis in patients with non-small cell lung cancer cases [32]. Similar results have been found in patients bearing pancreatic cancer. Previous investigations have proved that elevated NLR level correlated with unfavorable prognosis in patients who received curative resection [33] [34] [35] or bypass surgery [36]. The pretreatment NLR could predict survival for patients with advanced pancreatic cancer who received systematic chemotherapy as well [37]. In our present study, we found the elevated NLR level in newly diagnosed pancreatic cancer patients than in healthy subjects. Moreover, patients whose NLR values increased after chemotherapy, predicted worse outcomes. Univariate and multivariate analysis also confirmed that post/pre-chemotherapy NLR ratio is a prognostic marker affecting OS in pancreatic cancer, suggesting the application of NLR not only possesses diagnostic value but also be able to provide prognostic information in pancreatic cancers.

Platelets also play a crucial role in the development of tumors [23]. Researches have proved that platelets can promote angiogenesis, extracellular matrix degradation, release of growth factors and adhesion molecules, all of which are the basic parts for the proliferation and metastasis of tumors. Thus platelets are able to accelerate cancer development [10]. In the blood stream, covering with platelets helps circulating tumor cells (CTCs) to overcome the pressure in the blood, involving the attack from immune system and physical factors [38]. Besides the effect of platelet on the natural course of cancer, number and function of platelets could also be influenced by cancer. Increasing numbers of studies suggested that the proinflammatory cytokines released by tumors, like interleukin-1 (IL-1), interleukin-3 (IL-3), and interleukin-6 
(IL-6), can promote megakaryocyte proliferation, and lead to thrombocytosis gradually [10]. Therefore, an alliance of platelet and cancer provides a positive feedback on the progression of malignancies. In view of this, the platelet derived biomarkers present special potential.

By the combination of platelets and lymphocytes, PLR is thought to represent endogenous anticancer proinflammation and precoagulation in malignant tumors [9]. Besides, PLR is also assumed to be both a sensitive marker and a prognostic factor for breast cancers, ovarian cancers and colorectal cancers [39]. However, comparing with the value of NLR, many studies suggest that PLR levels are not qualified to be an independent prognostic factor for survival [10]. Our present findings indicated that, newly diagnosed pancreatic cancer patients have significantly higher PLR values comparing with the healthy controls, suggesting PLR could be a promising and easily available biomarker for the diagnosis of pancreatic cancer. It has been proved that cigarette smoke is a strong stimulator of pulmonary inflammation [40] [41]. In fact, elevated NLR and PLR levels have been observed in chronic obstructive pulmonary disease [42] [43] [44]. Therefore, the up-regulation of NLR and PLR could be correlated with both smoking history and cancer status, both of which are inflammation-related. Since both NLR and PLR are biomarkers reflecting systemic inflammation level, there is no surprise that patients with higher baseline NLR levels also possessed higher baseline PLR levels and vice versa. However, only NLR, but not PLR, associated with liver metastasis. Moreover, changes in PLR values after chemotherapy did not correlate with the response to treatment and overall survival, suggesting PLR is not as sensitive as NLR for the prognostic evaluation of pancreatic cancer, in consisting with a previous study [45].

Our study suggested that both NLR and PLR could be used in the diagnosis of pancreatic cancer. However, only NLR can be used to predict chemotherapy response and follow-up. In view of economic conditions in some areas of the world, this convenient, safe and lower cost biomarker may be beneficial for the patients with pancreatic cancer.

\section{MATERIALS AND METHODS}

\section{Subjects and inclusion criteria}

The study was conducted as a retrospective investigation of pancreatic cancer patients that had been referred to the First Affiliated Hospital of Soochow University (Jiangsu, China) between Jan 2007 and Mar 2015. Approval for the study was granted by the Medical Ethics Committees of the First Affiliated Hospital of Soochow University. Clinical and pathological records of all the patients participating in the study were reviewed periodically.
In total, 122 pancreatic cancer patients were recruited in this study. Patients had histologic or cytologic evidence of locally advanced or metastatic adenocarcinoma of the pancreas. Patient characteristics are detailed in Table 1 . The mean age of the 122 patients was 65 years (range, 32-80 years), and 74 patients were male and 48 were female. The inclusion criteria were as follows: (a) those with histologically or cytologically confirmed recurrent or metastatic gastric cancer; (b) age more than 18 years; (c) Karnofsky performance status (KPS) score of $\geq 70$; (d) those with a predicted survival of $\geq 3$ months; (e) either naive to anti-tumor treatment or the post-operative adjuvant chemotherapy was done at least six months after the last dose of chemotherapy; (f) in case of patients who were scheduled for radiotherapy on the target lesion, radiotherapy must have been finished for at least three months; (g) those with at least one measurable lesion (minimum $10 \mathrm{~mm} \times 10 \mathrm{~mm}$ on computed tomography (CT) or magnetic resonance imaging (MRI)); and (h) those who met the following laboratory criteria: white blood cells $(\mathrm{WBC}) \geq 4.0 \times 10^{9} / \mathrm{L}$; absolute neutrophil count (ANC) $\geq 1.5 \times 10^{9} / \mathrm{L}$; platelet $(\mathrm{PLT}) \geq 100 \times 10^{9} / \mathrm{L}$; Serum bilirubin $\leq$ upper limit of normal (ULN); alanine aminotransferase (ALT), aspartate aminotransferase (AST), and alkaline phosphatase $(\mathrm{ALP}) \leq \mathrm{ULN} \times 2.5$ (if without liver metastasis) or $\leq \mathrm{ULN} \times 5$ (if with liver metastasis); urea nitrogen $\leq \mathrm{ULN} \times 1.25$; and creatinine $\leq \mathrm{ULN} \times 1.25$. The staging of cancer was made according to tumornodulus-metastases (TNM) classification and classified through the American Joint Committee on Cancer (AJCC) recommendations, 7th edition [46]. Patients were followed regularly for 60 months. The prognostic analyses were performed regarding progression-free survival (PFS) and overall survival (OS). 30 age--sexes matched healthy subjects were also included into this study.

\section{Blood samples}

Peripheral venous blood $(5-7 \mathrm{ml})$ was collected into a sterile ethylenediaminetetraacetic acid (EDTA) tube. All blood samples were fasted and obtained between 6:30 and 7:30 a.m. in order to standardize the known impact of circulating hormones (circadian rhythm) on the number and subtype distribution of the various white blood cell indices. Hematological parameters were analyzed within $30 \mathrm{~min}$ after collection using a hematology analyser (Sysmex XE-2100; Sysmex, Kobe, Japan). Neutrophil $\left(10^{3} / \mu \mathrm{l}\right)$, lymphocyte $\left(10^{3} / \mu \mathrm{l}\right)$ and platelet $\left(10^{3} / \mu \mathrm{l}\right)$ counts and mean platelet volume (MPV) levels were recorded. NLR and PLR were calculated as the ratio of the neutrophils and platelets to lymphocytes, respectively. Mean value was used for NLR and PLR as normal distribution was absent. The patients were divided into two groups according to the mean value of NLR or PLR [(NLR low, $<3.81$ or NLR high, $\geq 3.81$; and PLR low, $<142.14$ or PLR high, $\geq 142.14$, respectively]. The 
post-/pre-operative ratios were defined as the rate of preoperative PLR or NLR values and the corresponding ones obtained one month after operation.

\section{Chemotherapy and evaluation}

In this study, most of the patients (98.4\%) underwent gemcitabine-based chemotherapy and a minority of the patients $(1.6 \%)$ underwent fluorine pyrimidines (fluorouracil, capecitabine, or S1 [tegafur, 5-chloro-2,4-dihydroxypyridine and oxonic acid]) treatment according to the newest edition of the National Comprehensive Cancer Network (NCCN) Guideline at the time. The regimen of gemcitabine alone was $1,000 \mathrm{mg} / \mathrm{m}^{2}$ ( 3 times per week followed by a 1-week rest for 6 cycles). Computed tomography (CT) scan was performed for the assessment of response every 2 months and evaluated according to the criteria of Response Evaluation Criteria in Solid Tumors (RECIST) 1.1 [47]. The responses to chemoradiotherapy including complete remission, regression, stable disease and disease progression.

\section{Follow-up}

We recorded responses to chemoradiotherapy including complete remission, regression, stable disease, and disease progression, and overall survival (OS). After first line chemotherapy, disease progression after chemoradiotherapy was defined as lack of response to chemoradiotherapy. In contrast, stable disease, complete response or disease regression after chemoradiotherapy was defined as response to chemoradiotherapy. Patients were regularly followed for 25 months. Survival time was measured from the date of chemoradiotherapy until death or last clinical evaluation. The prognostic analyses were performed regarding overall survival (OS). OS was defined as the time from the diagnosed date to death from any cause.

\section{Statistical analysis}

All statistical analyses were performed using SPSS 19.0 software (Chicago, USA). The associations between NLR or PLR levels and clinicopathologic features or chemotherapeutic efficacy were explored and assessed by the $\chi 2$ tests. For analysis of survival data, Kaplan-Meier curves were constructed, and statistical analysis was carried out using the $\log$ rank test. Multivariate Cox regression was performed for each outcome parameter, using a backwards elimination technique to derive a potentially suitable set of predictors. All values of $P<0.05$ were considered statistically significant.

\section{CONFLICTS OF INTEREST}

The authors declare no conflicts of interest.

\section{FUNDING}

This work was supported by grants from the National Natural Science Foundation of China (grant nos. 81472296, 81602091, 81402176, 81402093, 81272542, and 81200369), the Six Major Talent Peak Project of Jiangsu Province (grant no. 2015-WSN-022), The Project of Invigorating Health Care through Science, Technology and Education, Jiangsu Provincial Medical Youth Talent (grant no. QNRC2016709), the Science and Education for Health Foundation of Suzhou for Youth (grant no. kjxw2015003), the Science and Technology Project Foundation of Suzhou (grant nos. SYS201464 and SYS201504), and the Science and Technology Foundation of Suzhou Xiangcheng (grant nos. SZXC2012-70, XJ201538 and XJ201451).

\section{REFERENCES}

1. Tao M, Liu L, Shen M, Zhi Q, Gong FR, Zhou BP, Wu Y, Liu H, Chen K, Shen B, Wu MY, Shou LM, Li W. Inflammatory stimuli promote growth and invasion of pancreatic cancer cells through NF-kappaB pathway dependent repression of PP2Ac. Cell Cycle. 2016; 15:381-393.

2. Xu MD, Liu SL, Feng YZ, Liu Q, Shen M, Zhi Q, Liu Z, Gu DM, Yu J, Shou LM, Gong FR, Zhu Q, Duan W, et al. Genomic characteristics of pancreatic squamous cell carcinoma, an investigation by using high throughput sequencing after in-solution hybrid capture. Oncotarget. 2017; 8:14620-14635. https://doi.org/10.18632/ oncotarget. 14678 .

3. Gong FR, Wu MY, Shen M, Zhi Q, Xu ZK, Wang R, Wang WJ, Zong Y, Li ZL, Wu Y, Zhou BP, Chen K, Tao M, Li W. PP2A inhibitors arrest G2/M transition through JNK/ Sp1- dependent down-regulation of CDK1 and autophagydependent up-regulation of p21. Oncotarget. 2015; 6:1846918483. https://doi.org/10.18632/oncotarget.4063.

4. McMillan DC. Systemic inflammation, nutritional status and survival in patients with cancer. Curr Opin Clin Nutr Metab Care. 2009; 12:223-226.

5. Schreiber RD, Old LJ, Smyth MJ. Cancer immunoediting: integrating immunity's roles in cancer suppression and promotion. Science. 2011; 331:1565-1570.

6. Walsh SR, Cook EJ, Goulder F, Justin TA, Keeling NJ. Neutrophil-lymphocyte ratio as a prognostic factor in colorectal cancer. J Surg Oncol. 2005; 91:181-184.

7. Azab B, Bhatt VR, Phookan J, Murukutla S, Kohn N, Terjanian T, Widmann WD. Usefulness of the neutrophilto-lymphocyte ratio in predicting short- and long-term mortality in breast cancer patients. Ann Surg Oncol. 2012; 19:217-224.

8. Malik HZ, Prasad KR, Halazun KJ, Aldoori A, Al-Mukhtar A, Gomez D, Lodge JP, Toogood GJ. Preoperative prognostic score for predicting survival after hepatic 
resection for colorectal liver metastases. Ann Surg. 2007; 246:806-814.

9. Proctor MJ, Morrison DS, Talwar D, Balmer SM, Fletcher CD, O'Reilly DS, Foulis AK, Horgan PG, McMillan DC. A comparison of inflammation-based prognostic scores in patients with cancer. A Glasgow Inflammation Outcome Study. Eur J Cancer. 2011; 47:2633-2641.

10. Seretis C, Seretis F, Lagoudianakis E, Politou M, Gemenetzis G, Salemis NS. Enhancing the accuracy of platelet to lymphocyte ratio after adjustment for large platelet count: a pilot study in breast cancer patients. Int $\mathrm{J}$ Surg Oncol. 2012; 2012:653608.

11. Wu Y, Zhou BP. Inflammation: a driving force speeds cancer metastasis. Cell Cycle. 2009; 8:3267-3273.

12. Kim DK, Oh SY, Kwon HC, Lee S, Kwon KA, Kim BG, Kim SG, Kim SH, Jang JS, Kim MC, Kim KH, Han JY, Kim HJ. Clinical significances of preoperative serum interleukin-6 and C-reactive protein level in operable gastric cancer. BMC Cancer. 2009; 9:155.

13. Kilincalp S, Ekiz F, Basar O, Ayte MR, Coban S, Yilmaz B, Altinbas A, Basar N, Aktas B, Tuna Y, Erbis H, Ucar E, Erarslan E, Yuksel O. Mean platelet volume could be possible biomarker in early diagnosis and monitoring of gastric cancer. Platelets. 2014; 25:592-594.

14. Feig C, Gopinathan A, Neesse A, Chan DS, Cook N, Tuveson DA. The pancreas cancer microenvironment. Clin Cancer Res. 2012; 18:4266-4276.

15. Tassi E, Gavazzi F, Albarello L, Senyukov V, Longhi R, Dellabona P, Doglioni C, Braga M, Di Carlo V, Protti MP. Carcinoembryonic antigen-specific but not antiviral CD4+ T cell immunity is impaired in pancreatic carcinoma patients. J Immunol. 2008; 181:6595-6603.

16. Ebrahimi B, Tucker SL, Li D, Abbruzzese JL, Kurzrock R. Cytokines in pancreatic carcinoma: correlation with phenotypic characteristics and prognosis. Cancer. 2004; 101:2727-2736.

17. Fearon KC, Glass DJ, Guttridge DC. Cancer cachexia: mediators, signaling, and metabolic pathways. Cell Metabol. 2012; 16:153-166.

18. Mantovani A, Allavena P, Sica A, Balkwill F. Cancer-related inflammation. Nature. 2008; 454:436-444.

19. Stotz M, Gerger A, Eisner F, Szkandera J, Loibner H, Ress AL, Kornprat P, AlZoughbi W, Seggewies FS, Lackner C, Stojakovic T, Samonigg H, Hoefler G, Pichler M. Increased neutrophil-lymphocyte ratio is a poor prognostic factor in patients with primary operable and inoperable pancreatic cancer. Br J Cancer. 2013; 109:416-421.

20. Szkandera J, Gerger A, Liegl-Atzwanger B, Absenger G, Stotz M, Friesenbichler J, Trajanoski S, Stojakovic T, Eberhard K, Leithner A, Pichler M. The lymphocyte/ monocyte ratio predicts poor clinical outcome and improves the predictive accuracy in patients with soft tissue sarcomas. Int J Cancer. 2014; 135:362-370.
21. Yu SL, Xu LT, Qi Q, Geng YW, Chen H, Meng ZQ, Wang $\mathrm{P}$, Chen Z. Serum lactate dehydrogenase predicts prognosis and correlates with systemic inflammatory response in patients with advanced pancreatic cancer after gemcitabinebased chemotherapy. Sci Rep. 2017; 7:45194.

22. Lee Y, Kim SH, Han JY, Kim HT, Yun T, Lee JS. Early neutrophil-to-lymphocyte ratio reduction as a surrogate marker of prognosis in never smokers with advanced lung adenocarcinoma receiving gefitinib or standard chemotherapy as first-line therapy. J Cancer Res Clin Oncol. 2012; 138:2009-2016.

23. Kemal Y, Yucel I, Ekiz K, Demirag G, Yilmaz B, Teker F, Ozdemir M. Elevated serum neutrophil to lymphocyte and platelet to lymphocyte ratios could be useful in lung cancer diagnosis. Asian Pac J Cancer Prev. 2014; 15:2651-2654.

24. De Larco JE, Wuertz BR, Furcht LT. The potential role of neutrophils in promoting the metastatic phenotype of tumors releasing interleukin-8. Clin Cancer Res. 2004; 10:4895-4900.

25. Suzuki K, Kachala SS, Kadota K, Shen R, Mo Q, Beer DG, Rusch VW, Travis WD, Adusumilli PS. Prognostic immune markers in non-small cell lung cancer. Clin Cancer Res. 2011; 17:5247-5256.

26. Ohashi R, Takahashi K, Miura K, Ishiwata T, Sakuraba S, Fukuchi Y. Prognostic factors in patients with inoperable non-small cell lung cancer--an analysis of long-term survival patients. Gan To Kagaku Ryoho. 2006; 33:1595-1602.

27. Milne K, Alexander C, Webb JR, Sun W, Dillon K, Kalloger SE, Gilks CB, Clarke B, Kobel M, Nelson BH. Absolute lymphocyte count is associated with survival in ovarian cancer independent of tumor-infiltrating lymphocytes. J Transl Med. 2012; 10:33.

28. Kobayashi N, Usui S, Kikuchi S, Goto Y, Sakai M, Onizuka M, Sato Y. Preoperative lymphocyte count is an independent prognostic factor in node-negative non-small cell lung cancer. Lung Cancer. 2012; 75:223-227.

29. Kitayama J, Yasuda K, Kawai K, Sunami E, Nagawa H. Circulating lymphocyte number has a positive association with tumor response in neoadjuvant chemoradiotherapy for advanced rectal cancer. Radiat Oncol. 2010; 5:47.

30. Ding PR, An X, Zhang RX, Fang YJ, Li LR, Chen G, Wu XJ, Lu ZH, Lin JZ, Kong LH, Wan DS, Pan ZZ. Elevated preoperative neutrophil to lymphocyte ratio predicts risk of recurrence following curative resection for stage IIA colon cancer. Int J Colorectal Dis. 2010; 25:1427-1433.

31. Wang F, Liu ZY, Xia YY, Zhou C, Shen XM, Li XL, Han SG, Zheng Y, Mao ZQ, Gong FR, Tao M, Lian L, Li W. Changes in neutrophil/lymphocyte and platelet/ lymphocyte ratios after chemotherapy correlate with chemotherapy response and prediction of prognosis in patients with unresectable gastric cancer. Oncol Lett. 2015; 10:3411-3418. 
32. Akinci Ozyurek B, Sahin Ozdemirel T, Buyukyaylaci Ozden S, Erdogan Y, Kaplan B, Kaplan T. Prognostic value of the neutrophil to lymphocyte ratio (NLR) in lung cancer cases. Asian Pac J Cancer Prev. 2017; 18:1417-1421.

33. Garcea G, Ladwa N, Neal CP, Metcalfe MS, Dennison AR, Berry DP. Preoperative neutrophil-to-lymphocyte ratio (NLR) is associated with reduced disease-free survival following curative resection of pancreatic adenocarcinoma. World J Surg. 2011; 35:868-872.

34. Stevens L, Pathak S, Nunes QM, Pandanaboyana S, Macutkiewicz C, Smart N, Smith AM. Prognostic significance of pre-operative C-reactive protein and the neutrophil-lymphocyte ratio in resectable pancreatic cancer: a systematic review. HPB (Oxford). 2015; 17:285-291.

35. Cheng H, Luo G, Lu Y, Jin K, Guo M, Xu J, Long J, Liu L, Yu X, Liu C. The combination of systemic inflammationbased marker NLR and circulating regulatory $\mathrm{T}$ cells predicts the prognosis of resectable pancreatic cancer patients. Pancreatology. 2016; 16:1080-1084.

36. Sugiura T, Uesaka K, Kanemoto H, Mizuno T, Okamura Y. Elevated preoperative neutrophil-to-lymphocyte ratio as a predictor of survival after gastroenterostomy in patients with advanced pancreatic adenocarcinoma. Ann Surg Oncol. 2013; 20:4330-4337.

37. Luo G, Guo M, Liu Z, Xiao Z, Jin K, Long J, Liu L, Liu C, Xu J, Ni Q, Yu X. Blood neutrophil-lymphocyte ratio predicts survival in patients with advanced pancreatic cancer treated with chemotherapy. Ann Surg Oncol. 2015; 22:670-676.

38. Lian L, Li W, Li ZY, Mao YX, Zhang YT, Zhao YM, Chen K, Duan WM, Tao M. Inhibition of MCF-7 breast cancer cell-induced platelet aggregation using a combination of antiplatelet drugs. Oncol Lett. 2013; 5:675-680.

39. Yavuzcan A, Caglar M, Ustun Y, Dilbaz S, Ozdemir I, Yildiz E, Ozkara A, Kumru S. Evaluation of mean platelet volume, neutrophil/lymphocyte ratio and platelet/lymphocyte ratio in advanced stage endometriosis with endometrioma. J Turk Ger Gynecol Assoc. 2013; 14:210-215.

40. Tuder RM, Petrache I. Pathogenesis of chronic obstructive pulmonary disease. J Clin Invest. 2012; 122:2749-2755.

41. Brusselle GG, Joos GF, Bracke KR. New insights into the immunology of chronic obstructive pulmonary disease. Lancet. 2011; 378:1015-1026.

42. Rahimirad S, Ghaffary MR, Rahimirad MH, Rashidi F. Association between admission neutrophil to lymphocyte ratio and outcomes in patients with acute exacerbation of chronic obstructive pulmonary disease. Tuberk Toraks. 2017; 65:25-31.

43. Lee H, Um SJ, Kim YS, Kim DK, Jang AS, Choi HS, Kim YH, Kim TE, Yoo KH, Jung KS. Association of the neutrophil-to-lymphocyte ratio with lung function and exacerbations in patients with chronic obstructive pulmonary disease. PLoS One. 2016; 11:e0156511.

44. Chen G, Wu C, Luo Z, Teng Y, Mao S. Platelet-lymphocyte ratios: a potential marker for pulmonary tuberculosis diagnosis in COPD patients. Int J Chron Obstruct Pulmon Dis. 2016; 11:2737-2740.

45. Wang DS, Luo HY, Qiu MZ, Wang ZQ, Zhang DS, Wang $\mathrm{FH}, \mathrm{Li} \mathrm{YH}, \mathrm{Xu} \mathrm{RH}$. Comparison of the prognostic values of various inflammation based factors in patients with pancreatic cancer. Med Oncol. 2012; 29:3092-3100.

46. Edge SB, Compton CC. The American Joint Committee on Cancer: the 7th edition of the AJCC cancer staging manual and the future of TNM. Ann Surg Oncol. 2010; 17:1471-1474.

47. Eisenhauer EA, Therasse P, Bogaerts J, Schwartz LH, Sargent D, Ford R, Dancey J, Arbuck S, Gwyther S, Mooney M, Rubinstein L, Shankar L, Dodd L, et al. New response evaluation criteria in solid tumours: revised RECIST guideline (version 1.1). Eur J Cancer. 2009; 45:228-247. 